\title{
Cardiovascular disease in patients with chronic kidney disease
}

This article was published in the following Dove Press journal:

Vascular Health and Risk Management

25 August 2009

Number of times this article has been viewed

Julian Wright

Alastair Hutchison

Manchester Institute of Nephrology and Transplantation, Manchester Royal Infirmary, Manchester, UK
Correspondence: Alastair Hutchison Renal Dialysis Unit, The Royal Infirmary, Oxford Road, Manchester MI 3 9WL, UK Email alastair.hutchison@cmft.nhs.uk

\begin{abstract}
Patients with chronic kidney disease have a high burden of cardiovascular morbidity and mortality. The vast majority of patients with chronic kidney disease do not progress to end stage renal failure, but do have a significantly higher incidence of all cardiovascular co-morbidities. Traditional cardiovascular risk factors only partially account for this increased incidence of cardiovascular disease. In patients with kidney disease the basic biology underlying cardiovascular disease may be similar to that in patients without kidney disease, but it would seem many more risk factors are involved as a consequence of renal dysfunction. Although emphasis is placed on delaying the progression of chronic kidney disease, it must be appreciated that for many patients it is vital to address their cardiovascular risk factors at an early stage to prevent premature cardiovascular death. This review examines available epidemiological evidence, discusses common cardiovascular risk factors in patients with chronic kidney disease, and suggests possible treatment strategies. Potential areas for important research are also described.
\end{abstract}

Keywords: cardiovascular risk factors, chronic kidney disease, hypertension, diabetes

\section{Introduction}

Cardiovascular disease in patients with chronic kidney disease (CKD) is common and has major implications in terms of both human suffering and health economics. CKD is defined by the presence of kidney damage and level of kidney function - irrespective of the type of kidney disease. Among individuals with chronic kidney disease, the stages are defined by the level of kidney function, and CKD stage 3 comprises those with a stable, or gradually declining, estimated glomerular filtration rate (eGFR) 30 to $60 \mathrm{~mL} / \mathrm{min} / 1.73 \mathrm{~m}^{2}$.

Recognition of kidney disease has increased greatly in recent years, partly due to the widespread introduction of eGFR reporting, and partly as a result of the aging population which has an increasing prevalence of hypertension and diabetes - conditions in which minor kidney disease is very common, and clinically significant kidney disease is unfortunately often recognized too late to halt the relentless decline in kidney function. As a result of government initiatives UK primary care physicians are now keeping registers of patients with CKD in the same way that they have previously compiled them for diabetes and ischemic heart disease (IHD). Consequently the number of new patient referrals to nephrology clinics has soared over the last few years, and yet the key elements of hypertension and cardiovascular disease are often inadequately managed.

Once patients reach end stage kidney disease (CKD stage 5) and enter dialysis programs, they have an alarmingly high rate of cardiovascular death with those in 
the youngest age range of $<25$ years having equivalent cardiovascular mortality rates to 75 - to 85 -year-olds in the general population. ${ }^{1}$ Hence there is now increasing interest in the cardiovascular status of patients with earlier stages of CKD where efforts can be made to prevent cardiovascular disease occurring. Most patients with CKD do not suffer from symptoms of uremia, nor indeed die from kidney disease. The majority of patients with CKD die from cardiovascular disease, before their kidney dysfunction requires replacement therapy. In this review, we examine the evidence for increased cardiovascular disease in patients with CKD and suggest potential areas for research and treatment which may lessen the disease burden.

\section{Epidemiological evidence for increased cardiovascular disease in patients with CKD}

When compared to age-matched controls with normal kidney function, patients with CKD have markedly higher cardiovascular mortality which is multifactorial in origin ${ }^{2}$. Perhaps directly because of this high mortality, patients with severe CKD have largely been excluded from interventional trials aimed at reducing cardiovascular risk, including trials of angiotensin converting enzyme inhibitors (ACEi) and statins. Kidney disease is also a catabolic state and the syndrome of malnutrition, inflammation and atherosclerosis is prevalent in patients with kidney disease, particularly once they reach CKD stage 5 (eGFR $<15 \mathrm{~mL} / \mathrm{min}$ ), with constant activation of many acute phase proteins and cytokines. ${ }^{3}$ These observations mean that it is difficult to tease out the influence of one particular factor in the overall increased risk of cardiovascular morbidity and mortality in patients with CKD.

The NEOERICA study of computerized records in UK primary care has produced useful data demonstrating the influence of CKD on the prevalence of cardiovascular comorbidities. ${ }^{4}$ This key study established that the prevalence of IHD at approximately $25 \%$ for the patient population with CKD 3 to 5 (eGFR $<60 \mathrm{~mL} / \mathrm{min}$ ) was more than double that of patients without CKD. This marked increase in prevalence is also seen for congestive cardiac failure, peripheral vascular disease and cerebrovascular disease.

Once patients progress to eGFR $<45 \mathrm{~mL} / \mathrm{min}$ then cardiovascular disease burden is increased compared to individuals with more preserved renal function. ${ }^{5}$ The presence of any cardiovascular disease, be it congestive cardiac failure, IHD or left ventricular hypertrophy $(\mathrm{LVH})$, predicts a faster decline in kidney function when adjusted for baseline GFR. ${ }^{6}$ This suggests that cardiovascular co-morbidity is itself a risk factor for kidney functional decline most likely through an enhanced inflammatory process. The scale of the problem is exemplified by the fact that at the time of referral to a nephrologist approximately one third of patients with CKD already have evidence of IHD.

The NEOERICA study also demonstrated that greater than $50 \%$ of primary care patients of the age 85 years or older have CKD, although whether this is simply evidence of a normal aging process remains a controversial point. This might suggest that much CKD is clinically irrelevant as many such patients are not symptomatic and the use of cardiovascular risk reduction strategies in this age group has questionable benefits with regard to reduction in mortality and morbidity. Therefore the challenge currently facing nephrologists is to establish the clinical relevance of reduced kidney function in an individual patient. It may be that the key point is management of cardiovascular risk rather than management of reduced GFR.

For patients with CKD, long term population follow-up studies have shown that even for those with severe CKD (eGFR 15 to $30 \mathrm{~mL} / \mathrm{min}$ ), there is a much higher likelihood of death than of commencing dialysis (45.7\% vs $19.9 \%$ incidence respectively over 5 years). ${ }^{7}$ Yet in patients with severe CKD, more emphasis is often placed on preparation for kidney replacement therapy, including discussion of the differing dialysis modalities and kidney transplantation work up, than cardiovascular risk reduction. In patients whose kidney dysfunction is managed by primary care physicians, the standardized mortality rate for those with CKD under 60 years of age is much higher than for older patients. This leads to the conclusion that patients under the age of 60 with early CKD may have the most to gain from cardiovascular risk factor manipulation.

\section{Risk factors for cardiovascular disease in patients with CKD}

Patients with CKD have higher rates of cardiovascular morbidity and mortality than would be predicted by Framingham models of cardiovascular risk. ${ }^{8}$ There are many reasons for this including importantly, the confounding additional cardiovascular risk arising from malnutrition which occurs because kidney dysfunction leads to a catabolic state. This then promotes inflammation, a key promoter in the development of cardiovascular disease. ${ }^{9}$ There are also several paradoxical associations of traditional cardiovascular risk factors seen in patients with CKD. Once patients are on dialysis, a high body mass index leads to lower cardiovascular risk than a low body mass index in contradiction to general 
population studies. ${ }^{10}$ Another interesting association is the 'J-shaped curve' effect of both cholesterol and systolic blood pressure with cardiovascular morbidity and mortality which is observed in patients with CKD. ${ }^{11,12}$

The effect of statins and other cholesterol lowering agents in CKD is as yet uncertain. Studies of transplant patients given Fluvastatin failed to show an overall reduction of cardiovascular morbidity or mortality ${ }^{13,14}$ and in type 2 diabetes patients on hemodialysis, atorvastatin had no significant effect on cardiovascular death, non fatal myocardial infarction or stroke. ${ }^{15}$ Perhaps the cardiovascular risk of patients receiving kidney replacement therapy in the form of dialysis or transplantation is too high to show a clear benefit from cholesterol reduction, suggesting that it is in patients with earlier stages of CKD that the health benefits of cardiovascular risk reduction might be maximized.

The traditional risk factors for cardiovascular disease such as hypertension, dyslipidemia, diabetes and obesity are highly prevalent in CKD populations. However there are many other cardiovascular risk factors that are either 'uremia specific', or at least much more common in patients with CKD than in the general population. These factors include anemia, hyperparathyroidism, carnitine deficiency, hyperhomocysteinemia, low vitamin $\mathrm{C}$, high lipoprotein(a) levels and small apolipoprotein(a) size. To be of relevance clinically, treatment of individual risk factors must be able to be generalized to a diverse population. In addition, the risk factor must be modifiable with a demonstrable improvement in cardiovascular outcome. An example from nephrology where this has not been the case is hyperhomocysteinemia. Hyperhomocysteinemia is associated with poor cardiovascular prognosis in dialysis patients and the levels can be brought under control by supplementation with pyridoxine, vitamin B12 and folic acid. However such modification has not been shown to be reliable in reducing cardiovascular risk in kidney patients so that measurement of serum homocysteine, and treatment of elevated levels, is not widespread practice.

Anemia exerts a large influence over the pathogenesis of LVH, and correction with erythropoietin has been shown to reduce LVH. ${ }^{16}$ However this is also not straightforward. In studies where full correction of anemia has been compared with partial correction, the fully corrected group had a higher incidence of non fatal myocardial infarction than patients in whom anemia was only partially corrected. ${ }^{17}$ Malnutrition is another major factor in patients with $\mathrm{CKD}$, and can be the cause or effect of the chronic low grade inflammation frequently present in CKD. Both these cardiovascular risk factors are amenable to correction with careful assessment and treatment involving a multidisciplinary team comprising anemia specialist nurses and specialist renal dieticians.

\section{Diabetes and CKD}

Diabetic patients with CKD often present the most challenges for the nephrologist. Such patients have a higher incidence of cardiovascular co-morbidities such as IHD and peripheral vascular disease than any other patient group with CKD. The relationship between type 2 diabetes and hypertension is particularly strong with $79 \%$ of patients having either hypertension or abnormal circadian blood pressure cycles at the time of diagnosis. ${ }^{18}$ It is postulated that proteinuria reflects endothelial dysfunction so is a marker for increased risk of cardiovascular disease. Mortality in diabetes patients becomes strikingly high with the onset of proteinuria. General measures to reduce vascular risk in diabetes patients apply with the addition of good diabetes control. Trials of intervention for diabetes patients with respect to kidney impairment have focused on reducing conversion of normalbuminuria to microalbuminura, ${ }^{19,20}$ delaying the onset of diabetes nephropathy in type 2 diabetes patients with microalbuminura ${ }^{21,22}$ and delaying progressive kidney impairment once diabetes nephropathy has occurred, ${ }^{23,24}$ These interventions all involved blockade of the renin-angiotensin system (RAS). Interestingly, by the time patients have established diabetes nephropathy, intervention with RAS blocking agents has not been shown to alter cardiovascular risks overall, inferring that it is vital to commence treatment early in the pathway of kidney damage in patients with diabetes in order to affect their cardiovascular outcome. A post-hoc analysis of the Irbesartan in Diabetic Nephropathy Trial did show an overall mortality benefit for those patients with systolic blood pressure $<120 \mathrm{~mm} \mathrm{Hg}$ compared to those patients with higher systolic blood pressure. ${ }^{25}$

The Steno- 2 trial demonstrated a 50\% reduction in microvascular and microvascular complications in type 2 diabetes patients with microalbuminuira randomized to an intensive treatment arm with close attention paid to blood pressure, glycemic control, lipids and lifestyle measure; thus even in diabetes patients cardiovascular risk can be reduced with appropriate measures. ${ }^{26}$

\section{Hypertension and CKD}

As a potentially modifiable risk factor, the impact of hypertension on cardiovascular disease in patients with CKD is of great interest. As kidney impairment progresses there is increasing activation of the RAS in response to glomerular sclerosis and interstitial disease as well as 
fluid overload and increased arterial stiffness, all of which contribute to hypertension. Essential hypertension itself causes microvascular damage in the renal vascular bed causing kidney damage and hence, through RAS activation, exacerbating essential hypertension. Hypertension is known to alter renal physiological function with increased filtration fraction of sodium and increased renovascular resistance seen in hypertensive patients. ${ }^{27}$

Poor hypertension control clearly leads to increasing risk of cardiovascular morbidity and mortality and increasing risk of declining kidney function. A 'vicious circle' is created with worsening kidney function itself then contributing to hypertension. Hypertension is widespread among an otherwise healthy general population, especially in the elderly where approximately two thirds of people will be hypertensive. The majority of patients with CKD are hypertensive, with the prevalence increasing with increasing severity of CKD such that in the most severe stages of CKD (eGFR $<30 \mathrm{~mL} / \mathrm{min}$ ) over $90 \%$ of patients are hypertensive. ${ }^{3}$ As CKD progresses there is also increasing loss of the physiological nocturnal 'dip' in blood pressure, which is in itself a marker for the presence of $\mathrm{LVH}^{28}$

Studies have clearly shown a significant reduction in the rate of progression of CKD when hypertension is treated. Particularly this has been demonstrated in major studies investigating blockade of the RAS with ACEi in both proteinuric and nonproteinuric $\mathrm{CKD}^{29}$ and with angiotensin II receptor blockers. ${ }^{15,16}$ Many studies have demonstrated a reduction in the rate of progression of CKD with drugs blocking the RAS, compared to the same blood pressure reduction achieved with other antihypertensive regimens. This alludes to the importance of blocking angiotensin II which is a powerful an endothelial growth factor in the renal vascular bed, as well as the body's most powerful vasoconstrictor. There is not currently a full understanding of the pathophysiology of the effects of hypertension on the kidney in patients with CKD; however the major effect is likely to be a progressive increase in intra-renal vascular resistance which may precede any changes in kidney structure.

The Hypertension Detection and Follow Up Program followed over 10,000 general population patients over 5 years in a randomized controlled trial comparing usual antihypertensive care with a stepped program of drug interventions to achieve reduction in diastolic blood pressure of $10 \mathrm{~mm} \mathrm{Hg}$ (or $<90 \mathrm{~mm} \mathrm{Hg}$ in those patients whose baseline diastolic blood pressure was $\geq 100 \mathrm{~mm} \mathrm{Hg}$ ). As well as a $17 \%$ reduction in mortality, this trial established that the incidence of decline in kidney function was less in the stepped blood pressure treatment group than in other patients. The incidence of decline in kidney function was greatest in men and older adults as well as those with higher baseline diastolic blood pressure. The incidence of significant kidney impairment was low in this community based study, however an elevated serum creatinine was a potent independent risk factor for mortality. The lower rate of kidney impairment in the intensive blood pressure treatment group demonstrates the value of aggressive hypertension management in patients with CKD even in the early stages of kidney dysfunction. ${ }^{30}$ A smaller US study of patients with treated hypertension and initially normal serum creatinine levels also demonstrated that $15 \%$ of patients, went on to develop kidney dysfunction despite adequate blood pressure control. ${ }^{31}$

Altered kidney function is an adverse prognostic factor in populations with essential hypertension as well as in other patient populations, such as those with advanced congestive cardiac failure or a previous myocardial infarction. Even in the absence of a known primary kidney pathology, markers for kidney dysfunction (reduced eGFR, microalbuminuria and overt proteinuria) should be investigated in these populations because of the adverse prognostic implications of kidney dysfunction. In a study of patients with identified primary kidney diseases, elevated mean arterial blood pressure was independently correlated with a decrease in kidney function over time. ${ }^{32}$ Thus both in patients with essential hypertension and in those with primary kidney diseases and secondary hypertension, controlling blood pressure is an important means of reducing the rate of kidney decline as well as cardiovascular risk.

There is still debate about the ideal target level of blood pressure for patients with CKD. A meta-analysis of randomized controlled trials of ACEi showed that whilst increasing systolic blood pressure, above $120 \mathrm{~mm} \mathrm{Hg}$, proportionately increased the risk of progressive kidney dysfunction; increased risk was also seen with systolic blood pressures below $110 \mathrm{~mm} \mathrm{Hg}$. This finding can probably be explained by relative hypoperfusion of the kidney in patients with low systolic blood pressure secondary to heart failure. ${ }^{7}$ Such studies might lead clinicians to assume that there is a very narrow range of blood pressure which can be considered 'ideal' for patients with CKD. The current British Renal Association guidelines suggest a target blood pressure of $<130 / 80 \mathrm{~mm} \mathrm{Hg}$ to be acceptable in patients with stable kidney function and a target blood pressure of $<125 / 75 \mathrm{~mm} \mathrm{Hg}$ in patients with proteinuric kidney disease. ${ }^{33}$

In practice, it is often very difficult to achieve reasonable blood pressure control without resorting to polypharmacy, 
the side effects of which may not be acceptable to patients. In elderly patients who have a high incidence of postural hypotension, more individualized blood pressure targets may need to be set. General population studies such as the Hypertension Optimal Treatment study suggest that there is a clear advantage of minor reductions in blood pressure in terms of cardiovascular outcomes and physicians should therefore take heart that even small blood pressure reductions may have great benefit for individual patients over time. ${ }^{34}$ Similarly the Ramipril Efficacy in Nephropathy study demonstrated that adverse renal outcome (doubling of serum creatinine or the need for renal replacement therapy) was reduced with a minor reduction in blood pressure in the ramipril arm of the study compared to the placebo arm. ${ }^{20}$ Overall average blood pressure achieved in this study was suboptimal compared to recent targets, again suggesting that any blood pressure reduction is important even if targets are not achieved.

\section{Vascular calcification}

In the general population, atherosclerotic disease is manifest by intimal fibro-fatty plaque formation which may then become calcified. However, the medial layer of elastic arteries may also be involved in atherosclerosis with thickening and calcification occurring. This phenomenon, so called medial calcinosis, is associated with increased pulse wave velocity, elevated pulse pressure and systolic hypertension. Medial calcinosis is more common in diabetes patients, the elderly, and patients with CKD. It is known to be associated with increased cardiovascular mortality in patients with $\mathrm{CKD}^{35}$ and diabetes patients without $\mathrm{CKD} .{ }^{36}$

In elderly patients on dialysis there is evidence of greatly increased coronary artery calcification scores, as measured by electron beam computerized tomography, compared to age matched controls with no kidney impairment but angiographically proven coronary artery disease. ${ }^{37}$ Further studies in dialysis patients, have demonstrated increased mortality associated with increased coronary artery calcification, ${ }^{38}$ an association of coronary artery calcification with $\mathrm{LVH},{ }^{39}$ and an association of increased valvular calcification with mortality in patients with CKD. ${ }^{40}$

As with many aspects of cardiovascular disease in CKD patients there is a lack of knowledge regarding the process of vascular calcification in the early stages of CKD. Yet there is increasing evidence that abnormal bone mineralization which occurs early in the course of CKD, and vascular calcification are linked. Arterial calcification involves the transformation of vascular smooth muscle cells into bone forming osteoblast-like cells that express bone matrix proteins which are involved in arterial calcification. ${ }^{41}$

Several non-traditional risk factors for cardiovascular disease, which are highly prevalent in patients with CKD, can accelerate vascular calcification. These include hyperphosphatemia, hyperparathyroidism, hyperhomocysteinemia and increased levels of advanced glycosylation end products. Excess calcium can also induce smooth muscle cell mineralization in vitro. ${ }^{42}$

Both high and low bone turnover states occur in kidney patients. In high bone turn over, both osteoblast and osteoclast activity is high preventing adequate mineralization of the newly formed bone. In low bone turnover (adynamic bone) excessive hypercalcemia is common because the skeleton cannot act as an efficient 'sump' or buffer for calcium or phosphate. The situation is further complicated by calcium containing oral phosphate binders taken by patients in an effort to prevent gastro-intestinal phosphate absorption.

In dialysis patients there are links between decreased bone mineral density and increased coronary artery calcification. ${ }^{43}$ Peritoneal dialysis patients with biopsy proven adynamic bone disease had a $90 \%$ incidence of vascular calcification seen on plain $\mathrm{x}$-ray compared to $35 \%$ of those without adynamic bone disease. ${ }^{44}$

There is no doubt that both high and low bone turnover states carry risks for vascular calcification and that whist controlling hyperphosphatemia is of paramount importance in patients with CKD, a high calcium containing phosphate binder load is probably undesirable given our current state of knowledge. However, there is a paucity of research in this field in both dialysis patients and in the pre dialysis phase of CKD. Studies have shown that the progression of coronary calcification in patients new to hemodialysis is less for patients taking noncalcium-containing phosphate binders than for those taking calcium containing binders. ${ }^{45}$ Although a mortality benefit has yet to be proven in the overall hemodialysis population, a reduction in all-cause mortality for patients over 65 years of age has been demonstrated after 2 years sevelamer usage compared to patients on a calciumbased phosphate binder. ${ }^{46}$

Now that effective phosphate binders are available which do not contain calcium (sevelamer and lanthanum) and do not carry the risks associated with aluminium-based binders, studies can be performed to assess the risks of taking calcium containing versus noncalcium-containing binders on long-term cardiovascular outcomes in patients with early and severe CKD. 
Genetic pathways are also likely to be important in vascular calcification. Fetuin-A is a circulating inhibitor of vascular calcification and has been shown to be inversely correlated with coronary artery calcification in dialysis patients. ${ }^{47}$ Genetic variations in Fetuin-A may explain the variable vascular calcification seen in CKD patients. Loss of fibroblast growth factor 23 is also implicated in vascular calcification and is another example of genetic variation between individuals which may influence calcification in CKD.

\section{Left ventricular hypertrophy in CKD}

In patient populations on renal replacement therapy, LVH is an independent risk factor for cardiovascular related death which accounts for approximately $45 \%$ of mortality. Even in the early stages of kidney impairment, the prevalence of LVH is higher than in the general population. In a prospective echocardiographic study by Levin of patients attending a renal insufficiency clinic, LVH was present in $27 \%$ of patients with a creatinine clearance over $50 \mathrm{~mL} / \mathrm{min}$. In patients with clearances of 25 to $50 \mathrm{~mL} / \mathrm{min}$ and less than $25 \mathrm{~mL} / \mathrm{min}$, the prevalence of $\mathrm{LVH}$ was $31 \%$ and $45 \%$ respectively. These were significant differences between the functional groups, with the major predictors of LVH in this study being systolic hypertension and anemia. ${ }^{48}$

The cardiomyopathy of kidney failure results largely from pressure and volume overload, leading to changes which are, at least in the early phase, adaptive compensations in order to maintain adequate stroke volume. ${ }^{49}$ Pressure overload, resulting from hypertension and related to arterial stiffness and vascular calcification leads predominantly to concentric LVH. Volume overload could be assumed to cause predominantly left ventricular dilatation, resulting from salt and water overload, but anemia is another factor which might contribute to dilatation of the ventricle. The resulting cardiomyopathy leads to both left ventricular systolic and diastolic dysfunction. Systolic dysfunction in uremic patients has been well studied and it thought to be the result of premature myocyte death for which both IHD and the uremic environment are both predisposing factors.

Echocardiographic studies of patients at dialysis inception have revealed severe systolic dysfunction ( $\mathrm{LV}$ ejection fraction of $<25 \%$ ) in $15 \%$ of patients, with $74 \%$ of patients having LVH and $32 \%$ of patients demonstrating LV dilatation. ${ }^{50}$ Such LV abnormalities have been shown to have a direct influence on prognosis once patients begin dialysis. Practically such findings underlie the congestive cardiac failure suffered by dialysis patients which is then greatly exacerbated by volume overload and hypertension - both highly prevalent in this patient population. The advent of imaging techniques superior to echocardiography, such as cardiac magnetic resonance scanning has led to a new perspective on uremic cardiomyopathy. LVH has been demonstrated by late gadolinium enhancement on magnetic resonance imaging to be the predominant form of uremic cardiomyopathy with subendocardial fibrosis secondary to ischemia being an equally common finding. ${ }^{51}$

Most studies have been performed in patients already on renal replacement programs, but the evolution of such left ventricular morphological changes as CKD progresses remains largely unstudied. One might expect that early correction of anemia and strict hypertension control may slow down the development of LVH in patients with CKD. Such evidence already exists in dialysis patients with regards to the effect of increased hemoglobin levels, attained with the use of recombinant erythropoietin, leading to a decrease in LV mass and LV end diastolic volume, both surrogate markers for LVH. ${ }^{16,52}$ However no such robust data exist to date in patients with earlier stages of CKD.

\section{Potential areas for cardiovascular research in patients with CKD}

Currently, the nephrology referral guidelines for patients with moderate CKD (eGFR 30 to $60 \mathrm{~mL} / \mathrm{min}$ ) in the UK primary care setting rely on determining which patients have phenotypic features leading physicians to suspect that progressive kidney dysfunction is likely to occur. These features include hypertension which is poorly controlled despite the utilization of several classes of antihypertensive medications, progressive decrease in eGFR ( $>5 \mathrm{~mL} / \mathrm{min} /$ year), the presence of hematuria, significant proteinuria, normochromic anemia, electrolyte imbalances and a significant $(>15 \%)$ fall in eGFR after the introduction of drugs blocking the RAS. These features are associated with the need for further investigation or specialist treatments; however they do not accurately predict which patients will actually progress to requiring kidney replacement. Epidemiologically such patients are certainly at high risk of cardiovascular morbidity and mortality and this provides an opportunity to study the relationship between cardiovascular and kidney disease at an earlier stage than has previously been examined.

A key question is whether it is the same inflammatory process that drives both progression of kidney dysfunction and LVH. As angiotensin II is such a potent growth factor promoting LV hypertrophy and fibrosis as well as glomerular sclerosis all via the AT1 receptor, one might 
predict a common etiology. ${ }^{53}$ However whether advancing kidney disease itself promotes conditions under which there are enhanced effects of angiotensin II and other cytokines, remains the subject of current research. Candidate cytokines include interleukins 6 and 10, tumour necrosis factor, tissue growth factor beta, vascular endothelial growth factor (VEGF), C reactive protein and troponins. The complex interplay of these cytokines along with the persistent acute phase reaction and low level inflammation which occurs as kidney failure progresses in conjunction with malnutrition leading to heart failure, atherosclerosis and death is still to be fully mapped out.

In addition to the effects of cytokine load, it is clear that genotype is also important in determining the outcome of kidney dysfunction in patients with CKD. In general population studies, the homozygous deletion (D/D) ACE gene polymorphism is associated with high $\mathrm{LV}$ mass ${ }^{54}$ and is a marker of atherosclerotic complications and nephropathy in diabetes patients. ${ }^{55}$ Interestingly, meta-analyses of associations between cardiovascular and kidney disorders have not shown the D allele of the ACE gene to be associated with hypertension. The effect of ACE gene polymorphism on kidney outcome was demonstrated in 1996 with the deleterious effect of the D rather than the I allele of the ACE gene, with a steeper reciprocal creatinine versus time plot for patients homozygous for the deletion allele (D/D) compared to the insertion (I/I) allele. For patients with a baseline creatinine over $200 \mu \mathrm{mol} / \mathrm{L}$, the $\mathrm{I} / \mathrm{I}$ polymorphism was beneficial in terms of kidney outcomes. However, such an effect was only seen in patients with glomerular disease and not seen in patients with tubulo-interstitial disease. ${ }^{56}$ Once patients are on dialysis the $\mathrm{D}$ allele is also associated with increased cerebrovascular disease and carotid artery stenosis. ${ }^{57}$ Hence, although these effects are not fully understood, the ACE gene polymorphism clearly holds prognostic significance in terms of cardiovascular disease in patients with CKD.

Intuitively, other genetic polymorphisms must also be involved in determining vascular disease in patients with kidney impairment. One candidate, VEGF, is known to have certain polymorphisms associated with progression of kidney impairment in glomerulonephritis. ${ }^{58}$ VEGF is a platelet derived growth factor, which is an important signaling protein in angiogenesis and should be the subject of further research to delineate its effect on cardiovascular disease in renal patient populations.

Yet another area of interest is metabolomics and the information that might be gleaned from breakdown products of metabolism appearing in a patient's urine.
Certain substances such as Interleukin 18 and neutrophil gelatinase-associated lipocalcin are urinary markers of acute kidney injury in circumstances such as contrast induced nephropathy post coronary angiography ${ }^{59}$ At low levels they may also be markers of kidney ischemia in patients with CKD and allow profiling of patients based on risk of kidney or cardiovascular disease progression.

Genomics, metabolomics and the cytokine load might combine to produce a patient phenotype in which the risk of progressive cardiovascular disease in CKD might be high. Defining this phenotype is already in progress in the UK with the keeping of registers of patients with CKD stage 3 in primary care which will no doubt inform large-scale studies of progressive disease and in time interventional trials.

\section{Treatment of cardiovascular risk in patients with CKD}

In most large-scale cardiovascular intervention trials, patients with significant kidney impairment have been systematically excluded because of the high burden of cardiovascular co morbidity and mortality carried by this patient population. Hence most cardiovascular disease preventative treatment regimens in patients with CKD are currently based on trials in a nonrenal population.

Lifestyle measures such as regular exercise and a healthy diet are of importance and perhaps the long-term relationship between patient and physician in CKD lends itself to regular exhortation and encouragement in this direction. In particular salt restriction (target sodium intake $<100 \mathrm{mmol} /$ day) is certainly important in hypertensive patients or those with signs or symptoms of fluid overload. Smoking cessation is also of primary importance although the support for patients in the form of smoking cessation counsellors and clinics is often woefully lacking.

Hypertension may be the cause or effect of CKD. However, it is clear that poor blood pressure control leads to increased risk of progressive kidney impairment through the mechanisms of hyperfiltration and this is evidenced by proteinuria. Poor blood pressure control also increases risks of vascular disease in patients with CKD. In view of this, treatment should be aggressive, and strict targets aimed for $(<130 / 80 \mathrm{~mm} \mathrm{Hg}$ in patients without proteinuria and $<125 / 75 \mathrm{~mm} \mathrm{Hg}$ in patients with diabetes mellitus or proteinuria).

There is evidence for benefit of drugs that block the RAS that is disproportionately greater than, and in addition to, their effect on lowering blood pressure. However the evidence for this benefit in CKD with proteinuria, in both diabetes 
and nondiabetes subjects, cannot be generalized to patients without proteinuria. There are good theoretical reasons for thinking that dual blockade of angiotensin II with both ACEi and angiotensin II receptor blockade might be additionally beneficial. Such regimens have been shown to be useful in patients with LV dysfunction in general populations ${ }^{60,61}$ as well for enhanced proteinuria reduction in patients with proteinuric kidney disease of nondiabetic ${ }^{62}$ or diabetic origin. ${ }^{63}$ Treatment with dual blockade of the RAS must be carefully considered in light of the recent ONTARGET trial. ${ }^{64}$ The results of this trail are somewhat worrying with respect to patients with CKD as dual blockade of RAS led to worse renal outcomes.

If such regimens are employed, patients should be warned of the dangers of continuing such medications with intercurrent volume depleting illness such as diarrhea and vomiting. It is also important that kidney function is checked before treatment is commenced and within one to two weeks of starting medication to ensure that kidney function has not deteriorated significantly. A physiological drop in eGFR of up to $15 \%$ may be tolerated but in significant renal artery stenosis or critical renal perfusion in patients with heart failure, the drop in eGFR may be increased and preclude the use of such medications.

Often at least three antihypertensive medications with differing mechanisms are required to achieve adequate blood pressure control. Loop diuretics are particularly effective alongside salt and water restriction. The nondihydropyridine ring calcium channel blockers also have a place in the treatment of hypertension in patients with CKD. If further classes of drug are needed, beta blockers, centrally acting agents, alpha blockers, and vascular smooth muscle relaxants all have a place within multiple drug regimens.

As most of the large scale cholesterol lowering trials excluded patients with severe CKD, targets for cholesterol are not specific to CKD patients but are assumed to be the same as those for secondary prevention of cardiovascular disease - total cholesterol $<4.0 \mathrm{mmol} / \mathrm{L}$ and low-density lipoprotein cholesterol $<2.0 \mathrm{mmol} / \mathrm{L}$. In patients with end stage kidney failure both the 4D study (in diabetes hemodialysis patients) and ALERT study (in transplant patients) failed to show an overall benefit of statin therapy in reducing cardiovascular risk in patients with CKD. There is some evidence that hyperlipidemia promotes progression of kidney dysfunction, but there is no convincing evidence as yet that using statins to lower cholesterol reduces progression of kidney disease or reduces cardiovascular risk in patients with CKD. The ongoing SHARP study is examining cholesterol reduction with a combination of simvastatin and ezetimibe versus placebo and includes patients with $\mathrm{CKD}$, so hopefully there will be a sound evidence base in the near future.

Given the high risk of vascular risk carried by patients with CKD, primary prevention with an anti platelet agent might seem to be a good additional measure. There are potential conflicts for physicians treating a patient after a coronary stent has been placed who may require clopidogrel and aspirin in which the risks of bleeding from Tenckhoff catheter insertion to allow peritoneal dialysis and kidney transplant surgery may be considered unacceptable for elective surgery.

\section{Conclusion}

Cardiovascular disease and kidney disease are linked by a common etiology and together result in a very poor prognosis. There are many incompletely understood pathological processes involved, especially in the early stages of CKD during which the scope for successful intervention is huge and the benefits to the patient likely to be great. Physicians must be diligent to preview cardiovascular disease in patients with CKD and take appropriate measures to reduce patient risk, working with the patient to promote healthy living and compliance with prescribed medications. The place of specific prognostic investigations may also be considered. In one study, the presence and severity of renal dysfunction gave additional prognostic value over dobutamine stress echocardiography findings for cardiac death and events. ${ }^{65}$ The presence and extent of ischemia on stress echocardiography is an independent predictor of death in patients with $\mathrm{CKD}$, the effect of which remained even after patients had a renal transplant. ${ }^{66}$

This is an area demanding urgent and focused attention in an effort to break the deadly synergy between kidney and cardiovascular disease.

\section{Disclosures}

The authors declare no conflicts of interest.

\section{References}

1. Sarnak MJ, Levey AS. Epidemiology of cardiac disease in dialysis patients. Seminars in Nephrology. 1999;12:69-76.

2. Tesar V. [Cardiovascular complications in patients with chronic renal insufficiency and chronic kidney failure]. Vnitr Lek. 2003;49(5): 383-387.

3. Lindholm B, Wang T, Heimburger O, Bergstrom J. Influence of different treatments and schedules on the factors conditioning the nutritional status in dialysis patients. Nephrol Dial Transplant. 1998;13 Suppl 6:66-73.

4. de LS, Chan T, Stevens P, O’Donoghue D, Hague N, Dzregah B, et al. Identifying patients with chronic kidney disease from general practice computer records. Fam Pract. 2005;22(3):234-241. 
5. Parikh NI, Hwang SJ, Larson MG, Levy D, Fox CS. Chronic kidney disease as a predictor of cardiovascular disease (from the Framingham Heart Study). Am J Cardiol. 2008;102(1):47-53.

6. Levin A, Singer J, Thompson CR, Ross H, Lewis M. Prevalent left ventricular hypertrophy in the predialysis population: identifying opportunities for intervention. Am J Kidney Dis. 1996;27(3):347-354.

7. Keith DS, Nichols GA, Gullion CM, Brown JB, Smith DH. Longitudinal follow-up and outcomes among a population with chronic kidney disease in a large managed care organization. Arch Intern Med. 2004;164(6):659-663.

8. McClellan WM, Chertow GM. Beyond Framingham: cardiovascular risk profiling in ESRD. J Am Soc Nephrol. 2005;16(6):1539-1541.

9. Bergstrom J, Lindholm B. Malnutrition, cardiac disease, and mortality: an integrated point of view. Am J Kidney Dis. 1998;32(5):834-841.

10. de MR, Snijder MB, van der Sman-de Beer, Seidell JC, Boeschoten EW, Krediet RT, et al. Association between body mass index and mortality is similar in the hemodialysis population and the general population at high age and equal duration of follow-up. J Am Soc Nephrol. 2007;18(3):967-974.

11. Goldwasser P, Mittman N, Antignani A, Burrell D, Michel MA, Collier J, et al. Predictors of mortality in hemodialysis patients. $J$ Am Soc Nephrol. 1993;3(9):1613-1622.

12. Jafar TH, Stark PC, Schmid CH, Landa M, Maschio G, De Jong PE, et al. Progression of chronic kidney disease: the role of blood pressure control, proteinuria, and angiotensin-converting enzyme inhibition: a patientlevel meta-analysis. Ann Intern Med. 2003;139(4):244-252.

13. Fellstrom B, Holdaas H, Jardine AG, Holme I, Nyberg G, Fauchald P, et al. Effect of fluvastatin on renal end points in the Assessment of Lescol in Renal Transplant (ALERT) trial. Kidney Int. 2004;66(4):1549-1555.

14. Fellstrom B, Jardine AG, Soveri I, Cole E, Neumayer HH, Maes B, et al. Renal dysfunction is a strong and independent risk factor for mortality and cardiovascular complications in renal transplantation. Am J Transplant. 2005;5(8):1986-1991.

15. Wanner C, Krane V, Marz W, Olschewski M, Mann JF, Ruf G, et al. Atorvastatin in patients with type 2 diabetes mellitus undergoing hemodialysis. N Engl J Med. 2005;353(3):238-248.

16. Silberberg J, Racine N, Barre P, Sniderman AD. Regression of left ventricular hypertrophy in dialysis patients following correction of anemia with recombinant human erythropoietin. Can J Cardiol. 1990;6(1):1-4.

17. Besarab A, Bolton WK, Browne JK, Egrie JC, Nissenson AR, Okamoto DM, et al. The effects of normal as compared with low hematocrit values in patients with cardiac disease who are receiving hemodialysis and epoetin. N Engl J Med. 1998;339(9):584-590.

18. Keller CK, Bergis KH, Fliser D, Ritz E. Renal findings in patients with short-term type 2 diabetes. $J$ Am Soc Nephrol. 1996;7(12):2627-2635.

19. Ruggenenti P, Fassi A, Ilieva AP, Bruno S, Iliev IP, Brusegan V, et al. Preventing microalbuminuria in type 2 diabetes. $N$ Engl J Med. 2004;351(19):1941-1951.

20. Patel A, MacMahon S, Chalmers J, Neal B, Woodward M, Billot L, et al. Effects of a fixed combination of perindopril and indapamide on macrovascular and microvascular outcomes in patients with type 2 diabetes mellitus (the ADVANCE trial): a randomised controlled trial. Lancet. 2007;370(9590):829-840.

21. Makino H, Haneda M, Babazono T, Moriya T, Ito S, Iwamoto Y, et al. Prevention of transition from incipient to overt nephropathy with telmisartan in patients with type 2 diabetes. Diabetes Care. 2007;30(6):1577-1578.

22. Parving HH, Lehnert H, Brochner-Mortensen J, Gomis R, Andersen S, Arner P. The effect of irbesartan on the development of diabetic nephropathy in patients with type 2 diabetes. $N$ Engl J Med. 2001;345(12):870-878.

23. Brenner BM, Cooper ME, de ZD, Keane WF, Mitch WE, Parving HH, et al. Effects of losartan on renal and cardiovascular outcomes in patients with type 2 diabetes and nephropathy. $N$ Engl J Med. 2001;345(12): 861-869.
24. Lewis EJ, Hunsicker LG, Clarke WR, Berl T, Pohl MA, Lewis JB, et al. Renoprotective effect of the angiotensin-receptor antagonist irbesartan in patients with nephropathy due to type 2 diabetes. $N$ Engl $J$ Med. 2001;345(12):851-860.

25. Berl T, Hunsicker LG, Lewis JB, Pfeffer MA, Porush JG, Rouleau JL, et al. Impact of achieved blood pressure on cardiovascular outcomes in the Irbesartan Diabetic Nephropathy Trial. J Am Soc Nephrol. 2005;16(7):2170-2179.

26. Gaede PH, Jepsen PV, Larsen JN, Jensen GV, Parving HH, Pedersen OB. [The Steno-2 study. Intensive multifactorial intervention reduces the occurrence of cardiovascular disease in patients with type 2 diabetes]. Ugeskr Laeger. 2003;165(26):2658-2661.

27. Fliser D, Franek E, Joest M, Block S, Mutschler E, Ritz E. Renal function in the elderly: impact of hypertension and cardiac function. Kidney Int. 1997;51(4):1196-1204.

28. Farmer CK, Goldsmith DJ, Cox J, Dallyn P, Kingswood JC, Sharpstone P. An investigation of the effect of advancing uraemia, renal replacement therapy and renal transplantation on blood pressure diurnal variability. Nephrol Dial Transplant. 1997;12(11):2301-2307.

29. Randomised placebo-controlled trial of effect of ramipril on decline in glomerular filtration rate and risk of terminal renal failure in proteinuric, non-diabetic nephropathy. The GISEN Group (Gruppo Italiano di Studi Epidemiologici in Nefrologia). Lancet. 1997;349(9069):1857-1863.

30. Shulman NB, Ford CE, Hall WD, Blaufox MD, Simon D, Langford HG, et al. Prognostic value of serum creatinine and effect of treatment of hypertension on renal function. Results from the hypertension detection and follow-up program. The Hypertension Detection and Follow-up Program Cooperative Group. Hypertension. 1989;13(5 Suppl):I80-I93.

31. Rostand SG, Brown G, Kirk KA, Rutsky EA, Dustan HP. Renal insufficiency in treated essential hypertension. $N$ Engl $J$ Med. 1989;320(11):684-688.

32. Hannedouche T, Albouze G, Chauveau P, Lacour B, Jungers P. Effects of blood pressure and antihypertensive treatment on progression of advanced chronic renal failure. Am J Kidney Dis. 1993;21(5 Suppl 2): 131-137.

33. Renal Association: Treatments of adults and children with renal failure: standards and audit measures. London: Royal College of Physicians of London and the Renal Association; 2002.

34. Hansson L, Zanchetti A, Carruthers SG, Dahlof B, Elmfeldt D, Julius S, et al. Effects of intensive blood-pressure lowering and lowdose aspirin in patients with hypertension: principal results of the Hypertension Optimal Treatment (HOT) randomised trial. HOT Study Group. Lancet. 1998;351(9118):1755-1762.

35. London GM, Guerin AP, Marchais SJ, Metivier F, Pannier B, Adda H. Arterial media calcification in end-stage renal disease: impact on all-cause and cardiovascular mortality. Nephrol Dial Transplant. 2003;18(9):1731-1740.

36. Lehto S, Niskanen L, Suhonen M, Ronnemaa T, Laakso M. Medial artery calcification. A neglected harbinger of cardiovascular complications in non-insulin-dependent diabetes mellitus. Arterioscler Thromb Vasc Biol. 1996;16(8):978-983.

37. Ibels LS, Alfrey AC, Huffer WE, Craswell PW, Anderson JT, Weil R III. Arterial calcification and pathology in uremic patients undergoing dialysis. Am J Med. 1979;66(5):790-796.

38. Matsuoka M, Iseki K, Tamashiro M, Fujimoto N, Higa N, Touma T, et al. Impact of high coronary artery calcification score (CACS) on survival in patients on chronic hemodialysis. Clin Exp Nephrol. 2004;8(1):54-58.

39. Yildiz A, Memisoglu E, Oflaz H, Yazici H, Pusuroglu H, Akkaya V, et al. Atherosclerosis and vascular calcification are independent predictors of left ventricular hypertrophy in chronic haemodialysis patients. Nephrol Dial Transplant. 2005;20(4):760-767.

40. Wang AY, Wang M, Woo J, Lam CW, Li PK, Lui SF, et al. Cardiac valve calcification as an important predictor for all-cause mortality and cardiovascular mortality in long-term peritoneal dialysis patients: a prospective study. J Am Soc Nephrol. 2003;14(1):159-168. 
41. Giachelli CM, Bae N, Denhardt DT, Alpers CE, Schwartz SM. Osteopontin is elevated during neointima formation in rat arteries and is a novel component of artherosclerotic plaques. J Clin Invest. 1993;92:1686-1696.

42. Yang H, Curinga G, Giachelli CM. Elevated extracellular calcium levels induce smooth muscle cell matrix mineralization in vitro. Kidney Int. 2004;66(6):2293-2299.

43. Braun J, Oldendorf M, Moshage W. Electron beam computer tomography in the evaluation of cardiac calcification in chronic dialysis patients. Am J Kidney Dis. 1996;27:394-401.

44. Hutchison AJ, Whitehouse RW, Boulton HF, Adams JE, Mawer EB, Freemont TJ, et al. Correlation of bone histology with parathyroid hormone, vitamin D3, and radiology in end-stage renal disease. Kidney Int. 1993;44(5):1071-1077.

45. Block GA, Spiegel DM, Ehrlich J, Mehta R, Lindbergh J, Dreisbach A, et al. Effects of sevelamer and calcium on coronary artery calcification in patients new to hemodialysis. Kidney Int. 2005;68(4):1815-1824.

46. Suki WN, Zabaneh R, Cangiano JL, Reed J, Fischer D, Garrett L, et al. Effects of sevelamer and calcium-based phosphate binders on mortality in hemodialysis patients. Kidney Int. 2007;72(9):1130-1137.

47. Moe SM, Reslerova M, Ketteler M, O’Neill K, Duan D, Koczman J, et al. Role of calcification inhibitors in the pathogenesis of vascular calcification in chronic kidney disease (CKD). Kidney Int. 2005;67(6): 2295-2304.

48. Levin A. Clinical epidemiology of cardiovascular disease in chronic kidney disease prior to dialysis. Semin Dial. 2003;16(2):101-105.

49. London GM, Parfrey PS. Cardiac disease in chronic uremia: pathogenesis. Adv Ren Replace Ther. 1997;4(3):194-211.

50. Foley RN, Parfrey PS, Harnett JD, Kent GM, Martin CJ, Murray DC, et al. Clinical and echocardiographic disease in patients starting end-stage renal disease therapy. Kidney Int. 1995;47(1):186-192.

51. Mark PB, Johnston N, Groenning BA, Foster JE, Blyth KG, Martin TN, et al. Redefinition of uremic cardiomyopathy by contrast-enhanced cardiac magnetic resonance imaging. Kidney Int. 2006;69(10):1839-1845.

52. Pascual J, Teruel JL, Moya JL, Liano F, Jimenez-Mena M, Ortuno J. Regression of left ventricular hypertrophy after partial correction of anemia with erythropoietin in patients on hemodialysis: a prospective study. Clin Nephrol. 1991;35(6):280-287.

53. Willenheimer R, Dahlof B, Rydberg E, Erhardt L. AT1-receptor blockers in hypertension and heart failure: clinical experience and future directions. Eur Heart J. 1999;20(14):997-1008.

54. Iwai N, Ohmichi N, Nakamura Y, Kinoshita M. DD genotype of the angiotensin-converting enzyme gene is a risk factor for left ventricular hypertrophy. Circulation. 1994;90(6):2622-2628.
55. Staessen JA, Wang JG, Ginocchio G, Petrov V, Saavedra AP, Soubrier F, et al. The deletion/insertion polymorphism of the angiotensin converting enzyme gene and cardiovascular-renal risk. J Hypertens. 1997;15(12 Pt 2):1579-1592.

56. Mclaughlin KJ, Harden PN, Ueda S, Boulton-Jones JM, Connell JM, Jardine AG. The role of genetic polymorphisms of angiotensinconverting enzyme in the progression of renal diseases. Hypertension. 1996;28(5):912-915.

57. Losito A, Kalidas K, Santoni S, Ceccarelli L, Jeffery S. Polymorphism of renin-angiotensin system genes in dialysis patients - association with cerebrovascular disease. Nephrol Dial Transplant. 2002;17(12):2184-2188.

58. Summers AM, Coupes BM, Brennan MF, Ralph SA, Short CD, Brenchley PE. VEGF -460 genotype plays an important role in progression to chronic kidney disease stage 5. Nephrol Dial Transplant. 2005;20(11):2427-2432.

59. Ling W, Zhaohui N, Ben H, Leyi G, Jianping L, Huili D, et al. Urinary IL-18 and NGAL as Early Predictive Biomarkers in Contrast-Induced Nephropathy after Coronary Angiography. Nephron Clin Pract. 2008;108(3):c176-c181.

60. Pfeffer MA, Swedberg K, Granger CB, Held P, McMurray JJ, Michelson EL, et al. Effects of candesartan on mortality and morbidity in patients with chronic heart failure: the CHARM-Overall programme. Lancet. 2003;362(9386):759-766.

61. Cohn JN, Tognoni G. A randomized trial of the angiotensinreceptor blocker valsartan in chronic heart failure. $N$ Engl J Med. 2001;345(23):1667-1675.

62. Presne C, Mansour J, Makdassi R, Choukroun G, Fournier A. The COOPERATE trial. Lancet. 2003;361(9362):1054.

63. Mogensen CE, Neldam S, Tikkanen I, Oren S, Viskoper R, Watts RW, et al. Randomised controlled trial of dual blockade of renin-angiotensin system in patients with hypertension, microalbuminuria, and non-insulin dependent diabetes: the candesartan and lisinopril microalbuminuria (CALM) study. BMJ. 2000;321(7274):1440-1444.

64. Mann JF, Schmieder RE, McQueen M, Dyal L, Schumacher H, Pogue J, et al. Renal outcomes with telmisartan, ramipril, or both, in people at high vascular risk (the ONTARGET study): a multicentre, randomised, double-blind, controlled trial. Lancet. 2008;372(9638):547-553.

65. Karagiannis SE, Feringa $\mathrm{HH}$, Elhendy A, van DR, Chonchol M, Vidakovic R, et al. Prognostic significance of renal function in patients undergoing dobutamine stress echocardiography. Nephrol Dial Transplant. 2008;23(2):601-607.

66. Bergeron S, Hillis GS, Haugen EN, Oh JK, Bailey KR, Pellikka PA. Prognostic value of dobutamine stress echocardiography in patients with chronic kidney disease. Am Heart J. 2007;153(3):385-391.
Vascular Health and Risk Management

\section{Publish your work in this journal}

Vascular Health and Risk Management is an international, peerreviewed journal of therapeutics and risk management, focusing on concise rapid reporting of clinical studies on the processes involved in the maintenance of vascular health; the monitoring, prevention and treatment of vascular disease and its sequelae; and the involvement of

\section{Dovepress}

metabolic disorders, particularly diabetes. This journal is indexed on PubMed Central and MedLine. The manuscript management system is completely online and includes a very quick and fair peer-review system, which is all easy to use. Visit http://www.dovepress.com/ testimonials.php to read real quotes from published authors. 